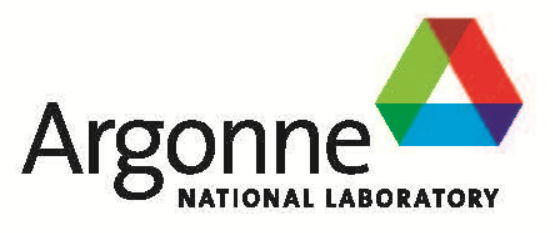

\title{
ANL/CSE-14/14
}

NATIONAL LABORATORY

\section{Verification of Column Design for Recovery of Mo from Low-Enriched Uranium Target using Irradiated Target Tracer Solution}

Chemical Sciences and Engineering Division 


\begin{abstract}
About Argonne National Laboratory
Argonne is a U.S. Department of Energy laboratory managed by UChicago Argonne, LLC under contract DE-AC02-06CH11357. The Laboratory's main facility is outside Chicago, at 9700 South Cass Avenue, Argonne, Illinois 60439. For information about Argonne and its pioneering science and technology programs, see www.anl.gov.
\end{abstract}

\title{
DOCUMENT AVAILABILITY
}

Online Access: U.S. Department of Energy (DOE) reports produced after 1991 and a growing number of pre-1991 documents are available free via DOE's SciTech Connect (http://www.osti.gov/scitech/)

Reports not in digital format may be purchased by the public from the National Technical Information Service (NTIS):

U.S. Department of Commerce

National Technical Information Service

5301 Shawnee Rd

Alexandra, VA 22312

unw.ntis.gov

Phone: (800) 553-NTIS (6847) or (703) 605-6000

Fax: (703) 605-6900

Email: orders@ntis.gov

Reports not in digital format are available to DOE and DOE contractors from the Office of Scientific and Technical Information (OST):

U.S. Department of Energy

Office of Scientific and Technical Information

P.O. Box 62

Oak Ridge, TN 37831-0062

unw.osti.gov

Phone: (865) 576-8401

Fax: (865) 576-5728

Email: reports@osti.gov

\begin{abstract}
Disclaimer
This report was prepared as an account of work sponsored by an agency of the United States Government. Neither the United States Government nor any agency thereof, nor UChicago Argonne, LLC, nor any of their employees or officers, makes any warranty, express or implied, or assumes any legal liability or responsibility for the accuracy, completeness, or usefulness of any information, apparatus, product, or process disclosed, or represents that its use would not infringe privately owned rights. Reference herein to any specific commercial product, process, or service by trade name, trademark, manufacturer, or otherwise, does not necessarily constitute or imply its endorsement, recommendation, or favoring by the United States Government or any agency thereof. The views and opinions of document authors expressed herein do not necessarily state or reflect those of the United States Government or any agency thereof, Argonne National Laboratory, or UChicago Argonne, LLC.
\end{abstract}




\section{Verification of Column Design for Recovery of Mo from Low-Enriched Uranium Target using Irradiated Target Tracer Solution}

by

Dominique C. Stepinski, Amanda J. Youker, and George F. Vandegrift

Chemical Sciences and Engineering Division, Argonne National Laboratory

prepared for

U.S. Department of Energy, National Nuclear Security Administration,

Office of Defense Nuclear Nonproliferation

August 2014 



\section{CONTENTS}

INTRODUCTION

2 RESULTS AND DISCUSSION

2.1 Design of Column Process Conditions for Alternative Target Solution Volumes . 3

2.2 Column Design Verification with Tracer Irradiated Target Solution .................... 4

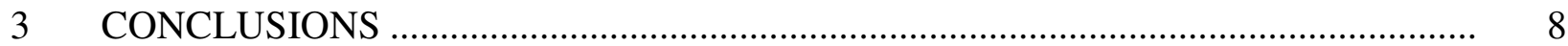

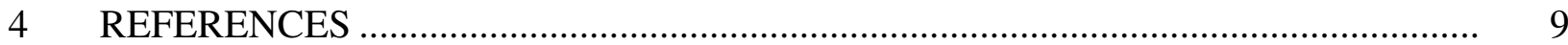

\section{FIGURES}

1 Langmuir Isotherm Data from Batch Tests and Langmuir Isotherm Model Fit

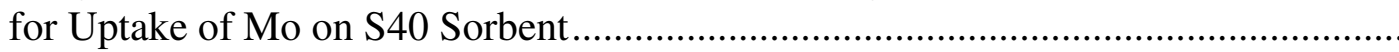

2 Estimation of Mo $D_{p}$ for S40 Sorbent from Breakthrough Experiment

\section{TABLES}

1 Process for Recovery of Mo from LEU Target Solution Using a 3.5 x $5 \mathrm{~cm}$

Column and S40 Sorbent

2 Design of Column Process Conditions for Alternative Target Solution Volumes ....... 5

3 Partitioning of Irradiated Target Solution Components between Process Streams ...... 6

4 Percent Partitioning of Irradiated Target Solution Components Normalized to $100 \%$

5 Partitioning of Decay Products between Process Streams 
This page intentionally left blank 


\section{VERIFICATION OF COLUMN DESIGN FOR RECOVERY OF MO FROM LOW-ENRICHED URANIUM TARGET USING IRRADIATED TARGET TRACER SOLUTION}

\section{INTRODUCTION}

The Conversion Program of the Global Threat Reduction Initiative (GTRI) is developing technology necessary to enable the conversion of civilian facilities using high-enriched uranium (HEU) to low-enriched uranium (LEU) for fuels and targets. The conversion of conventional HEU dispersion targets to LEU for the production of ${ }^{99}$ Mo requires approximately five times the uranium to maintain the ${ }^{99}$ Mo yield per target. Under GTRI, Argonne is developing two frontend options to the current processes to allow the use of LEU-foil targets. In both processes, the aim is to produce a product after the front end that will be compatible with current purification operations and that will, with the same number of targets irradiated, provide the same or higher yield of ${ }^{99} \mathrm{Mo}$ at the end of processing. The goal of the front-end process is to deliver a product solution that is of the same or higher Mo purity than the current solution and is of equal or better compatibility with current purification processes.

The two front-end processes under development as part of this project are: (1) the dissolution of irradiated LEU foil (up to $250 \mathrm{~g}$ in a single batch) and nickel fission recoil barrier in nitric acid at ambient pressure, followed by Mo recovery on a titania column, and (2) the electrochemical dissolution of LEU foil in a series of steps that produces an alkaline (basic) solution feed for ${ }^{99}$ Mo purification. This report describes results from recovery of Mo on a $3.5 \times 5 \mathrm{~cm}$ column containing the Sachtopore titania sorbent S40. The process is designed for a feed solution that contains $\sim 7 \mathrm{mM}$ Mo and $\sim 450 \mathrm{~g}-\mathrm{U} / \mathrm{L}$, and the nitric acid concentration after dissolution is $\sim 1 \mathrm{M}$. The volume of solution is assumed to be $500 \mathrm{~mL}$, and the pressure drop $(\Delta \mathrm{P})$ constraint is 0.8 atm based on use of vacuum/gravity fluid-transfer system.

The $3.5 \times 5 \mathrm{~cm}$ column was tested experimentally at $60{ }^{\circ} \mathrm{C}$ using a $1 \mathrm{M} \mathrm{H}^{+}$solution with $450 \mathrm{~g}-\mathrm{U} / \mathrm{L}, 7 \mathrm{mM}$ Mo, and tracer levels of Mo-99 (Stepinski et al., 2012). Loading velocity and column length were kept constant at $2.60 \mathrm{~cm} / \mathrm{min}$ and $5 \mathrm{~cm}$, respectively, while the column ID and solution volume were reduced to $1 \mathrm{~cm}$ and $40.8 \mathrm{~mL}$, respectively. Recovery of Mo was achieved with $0.1 \mathrm{M} \mathrm{NaOH}$ by counter-current stripping at $3 \mathrm{~cm} / \mathrm{min}$ and $25^{\circ} \mathrm{C}$. Also, $99.4 \pm$ $3 \%$ of Mo was loaded on the column, and $99.9 \pm 3 \%$ was recovered in strip fractions \#2 and \#3, six bed volumes (BV) each. The Mo-99 activity in the nitric acid wash (8 BV) and water wash (3 BV) fractions and strip fraction \#1 (4 BV) and \#4 (14 BV) was below detectable levels.

Based on the results, a Mo recovery process was designed by using a $3.5 \times 5 \mathrm{~cm}$ column with S40 sorbent (Table 1). In this process, $500 \mathrm{~mL}$ of solution is loaded at $60{ }^{\circ} \mathrm{C}$ in $20 \mathrm{~min}$ at $25 \mathrm{~mL} / \mathrm{min}$. The loading is followed by washing with $1 \mathrm{M} \mathrm{HNO}_{3}$ and $\mathrm{H}_{2} \mathrm{O}$ at $48.1 \mathrm{~mL} / \mathrm{min}$ and $25^{\circ} \mathrm{C}$. The Mo is stripped at $25^{\circ} \mathrm{C}$ with $770 \mathrm{~mL}$ of $0.1 \mathrm{M} \mathrm{NaOH}$ at a flow rate of $28.9 \mathrm{~mL} / \mathrm{min}$ for $26.7 \mathrm{~min}$. The total process time is $58 \mathrm{~min}$. The calculated pressure drops for the loading, washing, and strip are $0.35,0.43$, and 0.26 atm, respectively. 
TABLE 1 Process for Recovery of Mo from LEU Target Solution Using a $3.5 \times 5 \mathrm{~cm}$ Column and S40 Sorbent

\begin{tabular}{lccccc}
\hline \multicolumn{1}{c}{ Step } & $\begin{array}{c}\text { Loading } \\
\text { Time (min) }\end{array}$ & $\begin{array}{c}\text { Volume } \\
(\mathrm{mL})\end{array}$ & $\begin{array}{c}\text { Flow Rate } \\
(\mathrm{mL} / \mathrm{min})\end{array}$ & $\begin{array}{c}\text { Loading } \\
\text { Velocity } \\
(\mathrm{cm} / \mathrm{min})\end{array}$ & $\begin{array}{c}\text { Solution } \\
\text { Volume } \\
(\mathrm{BV})\end{array}$ \\
\hline & & & & & \\
Load & 20 & 500 & 25.0 & 2.60 & \\
Wash, $1 \mathrm{M} \mathrm{HNO}_{3}$ & 8 & 385 & 48.1 & 5 & 8 \\
Wash, $\mathrm{H}_{2} \mathrm{O}$ & 3 & 144 & 48.1 & 5 & 3 \\
Strip, $0.1 \mathrm{M} \mathrm{NaOH}$ & 26.7 & 770 & 28.9 & 3 & 16 \\
\hline
\end{tabular}

This report describes testing of the $3.5 \times 5 \mathrm{~cm}$ column design with $\mathrm{S} 40$ sorbent and $\sim 0.5 \mathrm{~L}$ of $1 \mathrm{M} \mathrm{H}^{+}$solution containing $450 \mathrm{~g} / \mathrm{L}$ uranyl nitrate and tracer irradiated target. The partitioning of fission products between process streams was monitored by gamma counting, and Mo yield under these conditions was determined. 


\section{RESULTS AND DISCUSSION}

\subsection{DESIGN OF COLUMN PROCESS CONDITIONS FOR ALTERNATIVE TARGET SOLUTION VOLUMES}

In a previous report (Stepinski et al., 2012), column designs for Sachtopore sorbent S40 with $40-\mu \mathrm{m}$ particle size, were determined using isotherms parameters for S80 sorbent. In this report we have determined Langmuir isotherm parameters for S40 sorbent and using VERSE simulation program (Purdue University) have estimated intraparticle diffusivity using the updated Langmuir isotherm parameters. Langmuir isotherm parameters for uptake of Mo from $450 \mathrm{~g}-\mathrm{U} / \mathrm{L}$ solution, $1 \mathrm{M} \mathrm{H}^{+}$solution on $\mathrm{S} 40$ sorbent were obtained using batch equilibrium data for samples equilibrated $24 \mathrm{~h}$ at $60{ }^{\circ} \mathrm{C}$ as described previously (Stepinski et al., 2012). Langmuir isotherm parameters were fitted using Origin 9.1 and are $\mathrm{a}=347, \mathrm{~b}=0.5 \mathrm{mM}^{-1}$ (Figure 1).

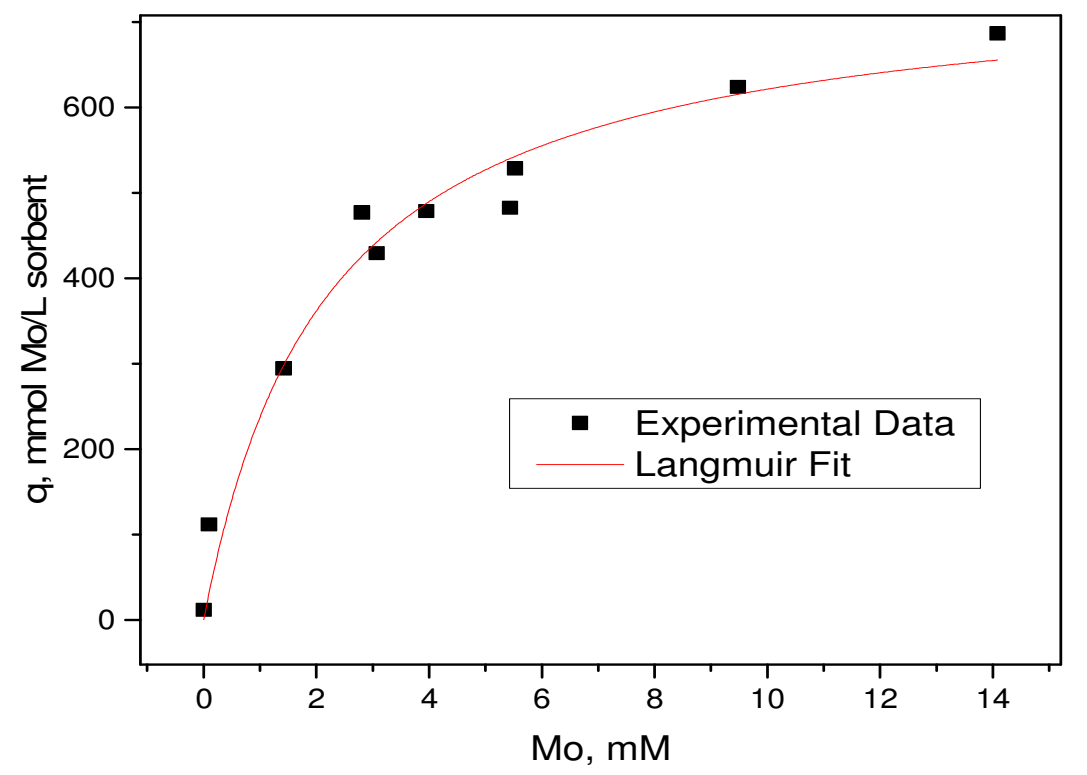

FIGURE 1 Langmuir Isotherm Data from Batch Tests and Langmuir Isotherm Model Fit for Uptake of Mo on S40 Sorbent

To estimate intraparticle diffusivity using the updated Langmuir isotherm parameters, the results of a breakthrough experiment obtained by loading $150 \mathrm{~mL}$ of $450 \mathrm{~g}-\mathrm{U} / \mathrm{L}, 6.5 \mathrm{mM}$ Mo and $1 \mathrm{M}$ $\mathrm{H}^{+}$solution onto $6.6 \times 35 \mathrm{~mm} \mathrm{~S} 40$ column at $3 \mathrm{~cm} / \mathrm{min}$ at $60{ }^{\circ} \mathrm{C}$, were simulated with VERSE. For the VERSE simulations the axial dispersion coefficient, $E_{b}$, was estimated with the Chung and Wen (1968) correlation. The mass transfer coefficient, $k_{f}$, was estimated with the Wilson and Geankoplis (1966) correlation. The Brownian diffusivity $\left(D_{\infty}\right)$ of $\mathrm{HMoO}_{4}{ }^{-}$in aqueous solution at $25{ }^{\circ} \mathrm{C}$ used in the simulations was $8.3 \mathrm{~cm}^{2} / \mathrm{min}$ (Marcus, 1997). The Stokes-Einstein equation was then used to correct the $D_{\infty}$ for solution viscosity and temperature effects. At 60 ${ }^{\circ} \mathrm{C}$, the viscosity of $450 \mathrm{~g}-\mathrm{U} / \mathrm{L} \mathrm{UO}_{2}\left(\mathrm{NO}_{3}\right)_{2}$ and $1 \mathrm{M} \mathrm{HNO}_{3}$ solution was calculated to be $1.40 \mathrm{cp}$, using a correlation listed in HW-57386 (Krigens, 1968). Therefore, at $60{ }^{\circ} \mathrm{C}, D_{\infty, M o}$ in $450 \mathrm{~g}-\mathrm{U} / \mathrm{L}$ $\mathrm{UO}_{2}\left(\mathrm{NO}_{3}\right)_{2}$ and $1 \mathrm{M} \mathrm{HNO}_{3}$ solution was calculated to be $5.51 \times 10^{-4} \mathrm{~cm}^{2} / \mathrm{min}$. The intraparticle 
diffusivity was estimated by comparing the experimental Mo breakthrough curve obtained by loading $150 \mathrm{~mL}$ of $450 \mathrm{~g}$-U/L, $6.5 \mathrm{mM}$ Mo and $1 \mathrm{M} \mathrm{H}^{+}$solution onto 6.6 x $35 \mathrm{~mm} \mathrm{~S} 40$ column at $3 \mathrm{~cm} / \mathrm{min}$ at $60{ }^{\circ} \mathrm{C}$, with VERSE simulations. In $450 \mathrm{~g}-\mathrm{U} / \mathrm{L} \mathrm{UO}_{2}\left(\mathrm{NO}_{3}\right)_{2}$ and $1 \mathrm{M} \mathrm{HNO}_{3}$ solution, the intraparticle diffusivities of Mo on S40, using $D_{\propto} / D_{p}=70$, was estimated to be $D_{p}=7.87 \mathrm{x}$ $10^{-6} \mathrm{~cm}^{2} / \mathrm{min}$ (Figure 2).

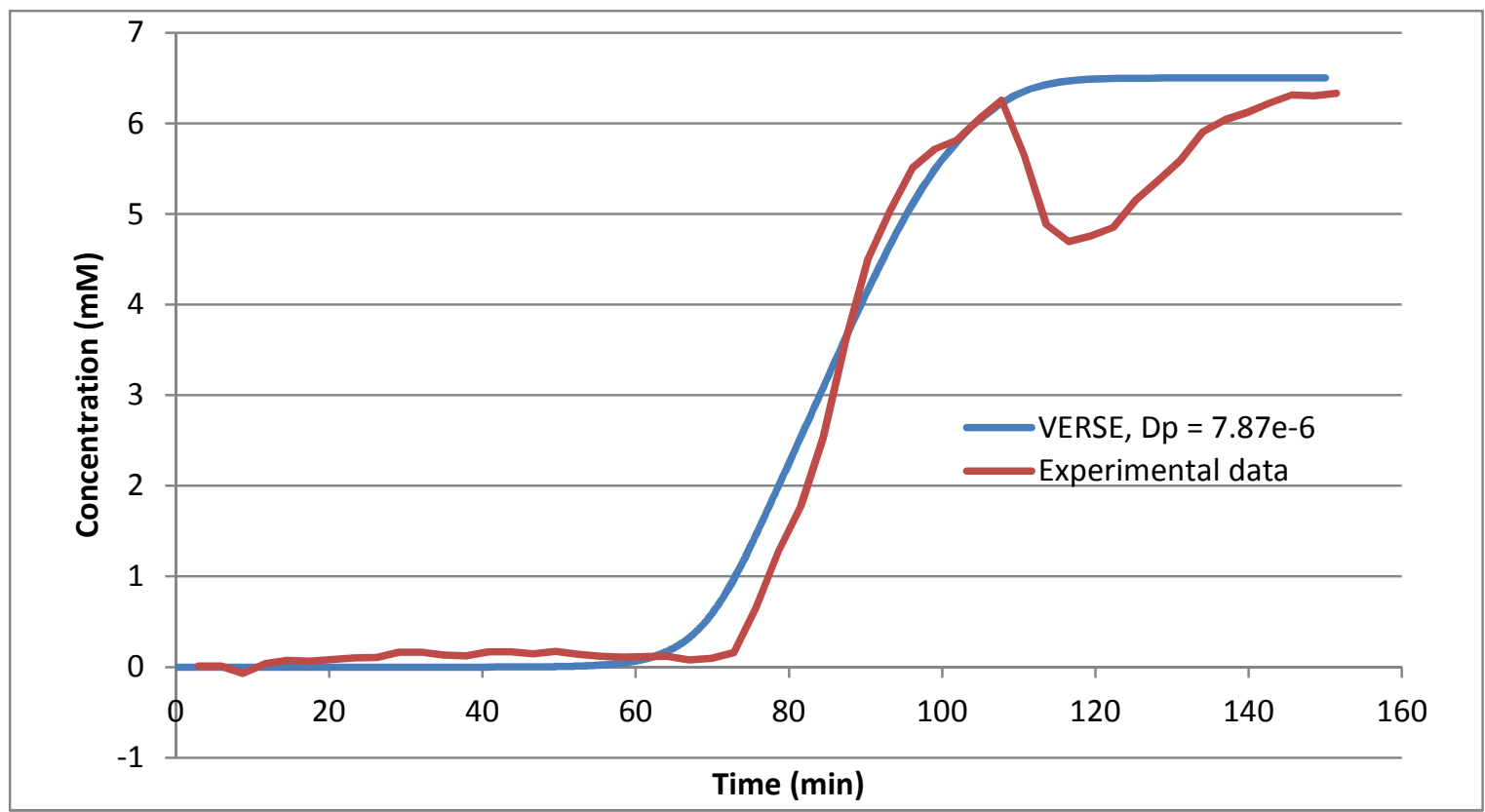

FIGURE 2 Estimation of Mo $D_{p}$ for S40 Sorbent from Breakthrough Experiment

To enable processing of a target solution obtained from dissolving $225 \mathrm{~g}$ uranium when the volume of solution varies between $0.5 \mathrm{~L}$ and $1 \mathrm{~L}$, we calculated alternative process conditions for loading Mo on a 3.5 x $5 \mathrm{~cm}$ column. Table 2 gives the solution properties, mass transfer parameters, and intraparticle diffusivity $(D p)$ values determined with VERSE simulations for various uranium solutions. The viscosities of the uranyl nitrate solution at $\mathrm{pH} 0$ were calculated with the correlation listed in HW-57386 (Krigens, 1968). The Stokes-Einstein equation was then used to correct the $D_{\infty}$ for solution viscosity and temperature effects. The Mo intraparticle diffusivities for S40 sorbent were calculated assuming $D_{\infty} / D_{p}=70$. VERSE was then used to calculate the mass transfer zone (MTZ). The results, shown in Table 2, indicate that larger solution volumes can be loaded at the same flow rate of $25 \mathrm{~mL} / \mathrm{min}$, since the combined effects of increased solution volume and higher intraparticle diffusivity have minimal effect on the MTZ.

\subsection{COLUMN DESIGN VERIFICATION WITH TRACER IRRADIATED TARGET SOLUTION}

The feed was prepared by dissolving irradiated LEU foil along with $250 \mathrm{~g}$ uranium in ambient pressure dissolver (Jerden et al., 2014). Stable $\mathrm{Mo}, \mathrm{Na}_{2} \mathrm{MoO}_{4}$, was added to the feed to increase the concentration of Mo to $5.4 \mathrm{mM}$, which is reflective of the amount of Mo in a fully irradiated target. The $\sim 650 \mathrm{~mL}(899 \mathrm{~g}, \sim 350 \mathrm{~g}-\mathrm{U} / \mathrm{L})$ of irradiated target solution was loaded on a 
$3.5 \times 5 \mathrm{~cm}$ column containing $63 \mathrm{~g}$ of $\mathrm{S} 40$ sorbent at $25 \mathrm{~mL} / \mathrm{min}$ and $60{ }^{\circ} \mathrm{C}$. The loading was followed by a $\sim 260 \mathrm{~mL} 1 \mathrm{M} \mathrm{HNO}_{3}$ wash and $75 \mathrm{~mL} \mathrm{H}_{2} \mathrm{O}$ wash. The Mo was stripped from the column with $0.1 \mathrm{M} \mathrm{NaOH}$ at $5 \mathrm{~cm} / \mathrm{min}$ and $25^{\circ} \mathrm{C}$. The strip was divided into four fractions containing $224,292,293$, and $120 \mathrm{~mL}$ of solution, respectively.

Tables 3 and 4 show partitioning of uranium and fission products between process streams. All activities were decay corrected to the start of Mo recovery process (on 5/1/2014 at $8 \mathrm{am})$. Table 3 shows the activities $(\mu \mathrm{Ci})$ of the irradiated solution components in process streams, and Table 4 indicates percent partitioning normalized to $100 \%$. The counting results indicate that $99.3 \%$ Mo was loaded on the column. Subsequently, $28.3 \%$ of Mo was in strip fraction \#2, and 70.1\% Mo in strip fraction \#3 (98.4\% Mo recovery). Strip fraction \#1 and \#4 contained $1 \%$ Mo. The Mo activities in the nitric acid wash and water wash fraction \#1 were below detection limits.

Furthermore, Tables 3 and 4 indicate that the majority of radionuclides, including uranium, iodine, alkaline metals ( $\mathrm{Ba}$ and $\mathrm{Sr}$ ), transition metals $(\mathrm{Cd}, \mathrm{Rh}$, and $\mathrm{Ru}$ ), and lanthanides (Ce and $\mathrm{Nd}$ ) partition into the eluent and nitric acid wash streams. Only Sb was found in the Mo

TABLE 2 Design of Column Process Conditions for Alternative Target Solution Volumes

\begin{tabular}{|c|c|c|c|c|c|c|c|c|c|c|c|c|c|c|}
\hline $\begin{array}{l}\text { U conc. } \\
\text { (gU/L) }\end{array}$ & $\begin{array}{l}\text { Volume } \\
(\mathrm{mL})\end{array}$ & $\begin{array}{l}\text { Mo } \\
\text { (mM) }\end{array}$ & $\mathrm{T}\left({ }^{\circ} \mathrm{C}\right)$ & $\begin{array}{l}\text { viscosity } \\
\text { (cp) }\end{array}$ & $\begin{array}{l}\text { density } \\
(\mathrm{g} / \mathrm{mL})\end{array}$ & $\begin{array}{c}D_{M o} \\
\left(\mathrm{~cm}^{2} / \mathrm{min}\right)\end{array}$ & $\begin{array}{c}\mathrm{Dp} \\
\left(\mathrm{cm}^{2} / \mathrm{min}\right)\end{array}$ & $\mathrm{ID}(\mathrm{cm})$ & $\begin{array}{l}\text { flowrate } \\
\text { (mL/min) }\end{array}$ & $\begin{array}{l}\text { time } \\
(\min )\end{array}$ & $\begin{array}{l}\text { MTZ } \\
(\mathrm{cm})\end{array}$ & $\mathrm{U}(\mathrm{M})$ & $\begin{array}{l}\mathrm{NO}_{3} \\
\text { (M) }\end{array}$ & $\mathrm{L}(\mathrm{cm})$ \\
\hline 450 & 500 & 7 & 60 & 1.49 & 1.6204 & 5.51E-04 & 7.87E-06 & 3.5 & 25 & 20 & 1.90 & 1.89 & 4.78 & 5 \\
\hline 409 & 550 & 6.4 & 60 & 1.34 & 1.5650 & $6.13 \mathrm{E}-04$ & $8.75 \mathrm{E}-06$ & 3.5 & 25 & 22 & 1.88 & 1.72 & 4.44 & 5 \\
\hline 375 & 600 & 5.8 & 60 & 1.22 & 1.5189 & $6.73 \mathrm{E}-04$ & $9.61 \mathrm{E}-06$ & 3.5 & 25 & 24 & 1.87 & 1.58 & 4.15 & 5 \\
\hline 346 & 650 & 5.4 & 60 & 1.13 & 1.4798 & $7.26 \mathrm{E}-04$ & $1.04 \mathrm{E}-05$ & 3.5 & 25 & 26 & 1.87 & 1.45 & 3.91 & 5 \\
\hline 321 & 700 & 5.0 & 60 & 1.06 & 1.4464 & 7.74E-04 & 1.11E-05 & 3.5 & 25 & 28 & 1.85 & 1.35 & 3.70 & 5 \\
\hline 300 & 750 & 4.7 & 60 & 1 & 1.4174 & $8.21 \mathrm{E}-04$ & 1.17E-05 & 3.5 & 25 & 30 & 1.87 & 1.26 & 3.52 & 5 \\
\hline 281 & 800 & 4.4 & 60 & 0.95 & 1.3920 & $8.64 \mathrm{E}-04$ & $1.23 \mathrm{E}-05$ & 3.5 & 25 & 32 & 1.88 & 1.18 & 3.36 & 5 \\
\hline 265 & 850 & 4.1 & 60 & 0.92 & 1.3696 & 8.92E-04 & $1.27 \mathrm{E}-05$ & 3.5 & 25 & 34 & 1.90 & 1.11 & 3.22 & 5 \\
\hline 250 & 900 & 3.9 & 60 & 0.88 & 1.3497 & $9.33 \mathrm{E}-04$ & $1.33 \mathrm{E}-05$ & 3.5 & 25 & 36 & 1.92 & 1.05 & 3.10 & 5 \\
\hline 237 & 950 & 3.7 & 60 & 0.85 & 1.3319 & $9.66 \mathrm{E}-04$ & $1.38 \mathrm{E}-05$ & 3.5 & 25 & 38 & 1.93 & 1.00 & 2.99 & 5 \\
\hline 225 & 1000 & 3.5 & 60 & 0.83 & 1.3158 & $9.89 \mathrm{E}-04$ & $1.41 \mathrm{E}-05$ & 3.5 & 25 & 40 & 1.95 & 0.95 & 2.89 & 5 \\
\hline
\end{tabular}


TABLE 3 Partitioning of Irradiated Target Solution Components between Process Streams

\begin{tabular}{|c|c|c|c|c|c|c|c|c|c|c|c|}
\hline Isotope & $\begin{array}{c}\text { Half-life } \\
\text { (days) }\end{array}$ & $\begin{array}{l}\text { Feed } \\
(\mu \mathrm{Ci})\end{array}$ & $\begin{array}{c}\text { Eluent } \\
(\mu \mathrm{Ci})\end{array}$ & $\begin{array}{c}1 \mathrm{M} \mathrm{HNO}_{3} \\
\text { Wash } \\
(\mu \mathrm{Ci})\end{array}$ & $\begin{array}{c}\mathrm{H}_{2} \mathrm{O} \\
\text { Wash } \\
(\mu \mathrm{Ci})\end{array}$ & $\begin{array}{c}\text { Strip } \\
\text { fraction } 1 \\
(\mu \mathrm{Ci})\end{array}$ & $\begin{array}{c}\text { Strip } \\
\text { fraction } 2 \\
(\mu \mathrm{Ci})\end{array}$ & $\begin{array}{c}\text { Strip } \\
\text { fraction } 3 \\
(\mu \mathrm{Ci})\end{array}$ & $\begin{array}{c}\text { Strip } \\
\text { fraction } 4 \\
(\mu \mathrm{Ci})\end{array}$ & $\begin{array}{c}\text { Sorbent } \\
(\mu \mathrm{Ci})\end{array}$ & $\begin{array}{c}\text { Mass } \\
\text { Balance } \\
(\%)\end{array}$ \\
\hline Ba-140 & 12.79 & $1.1 \mathrm{E}+01$ & $8.3 \mathrm{E}+00$ & $1.1 \mathrm{E}+00$ & - & - & - & - & - & - & $8.5 \mathrm{E}+01$ \\
\hline Ce-141 & 32.28 & $3.3 \mathrm{E}+00$ & 2.7E+00 & 3.7E-01 & - & - & - & - & - & - & $9.1 \mathrm{E}+01$ \\
\hline Ce-143 & 1.40 & $3.3 \mathrm{E}+01$ & $2.5 \mathrm{E}+01$ & $3.2 \mathrm{E}+00$ & - & - & - & - & - & - & $8.7 \mathrm{E}+01$ \\
\hline $\mathrm{I}-133$ & 0.87 & 3. $3 \mathrm{E}+01$ & $2.5 \mathrm{E}+01$ & $2.9 \mathrm{E}+00$ & $2.8 \mathrm{E}-03$ & 1.1E-02 & $1.0 \mathrm{E}-01$ & $1.5 \mathrm{E}-01$ & - & - & $8.7 E+01$ \\
\hline I-135 & 0.27 & 7.7E+00 & $8.3 \mathrm{E}+00$ & 7.0E-01 & - & - & - & - & - & - & $1.2 \mathrm{E}+02$ \\
\hline Mo-99 & 2.74 & $2.4 \mathrm{E}+01$ & $1.4 \mathrm{E}-01$ & - & - & 4.3E-02 & $6.2 \mathrm{E}+00$ & $1.5 \mathrm{E}+01$ & 1.7E-01 & - & $9.1 \mathrm{E}+01$ \\
\hline $\mathrm{Nd}-147$ & 11.06 & $5.1 \mathrm{E}+00$ & $4.5 \mathrm{E}+00$ & $9.5 \mathrm{E}-01$ & - & - & $9.2 \mathrm{E}-03$ & - & - & - & $1.1 \mathrm{E}+02$ \\
\hline Pm-151 & 1.18 & $2.2 \mathrm{E}+00$ & $1.8 \mathrm{E}+00$ & $2.4 \mathrm{E}-01$ & - & - & - & - & - & - & $9.1 \mathrm{E}+01$ \\
\hline Rh-105 & 1.47 & $7.5 \mathrm{E}+00$ & $6.4 \mathrm{E}+00$ & 7.0E-01 & - & - & - & - & - & - & $9.5 \mathrm{E}+01$ \\
\hline Ru-103 & 39.35 & $1.6 \mathrm{E}+00$ & $1.2 \mathrm{E}+00$ & 1.6E-01 & 1.5E-04 & - & $4.0 \mathrm{E}-03$ & $3.5 \mathrm{E}-03$ & 7.4E-04 & - & $8.4 \mathrm{E}+01$ \\
\hline Sb-127 & 3.85 & $6.8 \mathrm{E}-01$ & $8.8 \mathrm{E}-02$ & $1.2 \mathrm{E}-01$ & - & 3.2E-01 & - & - & - & $8.8 \mathrm{E}-03$ & $8.0 \mathrm{E}+01$ \\
\hline Sm-153 & 1.93 & $1.0 \mathrm{E}+01$ & $8.6 \mathrm{E}+00$ & $1.0 \mathrm{E}+00$ & $2.4 \mathrm{E}-03$ & 3.4E-03 & - & - & - & - & $9.4 E+01$ \\
\hline Sr-91 & 0.40 & $1.4 \mathrm{E}+01$ & $1.3 \mathrm{E}+01$ & $1.6 \mathrm{E}+00$ & 5.7E-04 & - & - & - & - & - & $1.0 \mathrm{E}+02$ \\
\hline Te-132 & 3.25 & $1.8 \mathrm{E}+01$ & $1.8 \mathrm{E}+00$ & 2.7E-01 & 5.7E-04 & 9.7E-03 & $5.9 \mathrm{E}-03$ & $5.8 \mathrm{E}-03$ & 1.0E-02 & 1.6E-01 & $1.3 \mathrm{E}+01$ \\
\hline U-237 & 6.75 & $1.5 \mathrm{E}+01$ & 8.7E+00 & $1.4 \mathrm{E}+00$ & - & - & - & - & - & - & $6.9 \mathrm{E}+01$ \\
\hline Y-93 & 0.46 & $2.6 \mathrm{E}+01$ & $2.8 \mathrm{E}+01$ & $0.0 \mathrm{E}+00$ & - & - & - & - & - & - & $1.1 \mathrm{E}+02$ \\
\hline $\mathrm{Zr}-95$ & 64.40 & $2.1 \mathrm{E}+00$ & $1.9 \mathrm{E}+00$ & - & - & $4.8 \mathrm{E}-03$ & 3.6E-03 & 1.6E-03 & - & 2.7E-02 & $9.2 \mathrm{E}+01$ \\
\hline
\end{tabular}

product stream $(\sim 60 \%$ in strip fraction \#1). Other irradiated target components such as Ru, I, Te, and $\mathrm{Zr}$ partitioned $<\% 1$ into the Mo strip fractions \#1-3. Fractions of Sb (1.6\%), Te (7.1\%), and $\mathrm{Zr}(1.4 \%)$ remained on the sorbent.

Table 5 shows the activities of I-131 and La-140, which are growing in from their respective parents, Te-131m and Ba-140; therefore, their activities are not decay-corrected. The activities of these isotopes were determined $\sim 24 \mathrm{~h}$ after the column experiment. As with other irradiated solution components, Te and $\mathrm{Ba}$ and their daughter isotopes mainly partition in the eluent and acid-wash process streams. 
TABLE 4 Percent Partitioning of Irradiated Target Solution Components Normalized to 100\%

\begin{tabular}{|c|c|c|c|c|c|c|c|c|c|c|}
\hline Isotope & $\begin{array}{c}\text { Half-life } \\
\text { (days) }\end{array}$ & $\begin{array}{c}\text { Eluent } \\
(\%)\end{array}$ & $\begin{array}{c}1 \mathrm{M} \mathrm{HNO}_{3} \\
\text { Wash } \\
(\%)\end{array}$ & $\begin{array}{c}\mathrm{H}_{2} \mathrm{O} \\
\text { Wash } \\
(\%)\end{array}$ & $\begin{array}{c}\text { Strip } \\
\text { fraction } 1 \\
(\%)\end{array}$ & $\begin{array}{c}\text { Strip } \\
\text { fraction } 2 \\
(\%)\end{array}$ & $\begin{array}{c}\text { Strip } \\
\text { fraction } 3 \\
(\%)\end{array}$ & $\begin{array}{c}\text { Strip } \\
\text { fraction } 4 \\
(\%)\end{array}$ & $\begin{array}{c}\text { Sorbent } \\
(\%)\end{array}$ & $\begin{array}{c}\text { Mass } \\
\text { Balance } \\
(\%)\end{array}$ \\
\hline Ba-140 & 12.79 & 88.2 & 11.8 & - & - & - & - & - & - & 85.1 \\
\hline Ce-141 & 32.28 & 87.9 & 12.1 & - & - & - & - & - & - & 91.4 \\
\hline Ce-143 & 1.404 & 88.8 & 11.2 & - & - & - & - & - & - & 86.9 \\
\hline I-133 & 0.867083 & 89.0 & 10.1 & 0.0 & 0.0 & 0.4 & 0.5 & - & - & 87.3 \\
\hline I-135 & 0.274167 & 92.2 & 7.8 & - & - & - & - & - & - & 116.0 \\
\hline Mo-99 & 2.743056 & 0.7 & - & - & 0.2 & 28.3 & 70.1 & 0.8 & - & 91.3 \\
\hline $\mathrm{Nd}-147$ & 11.06 & 82.4 & 17.5 & - & - & 0.2 & - & - & - & 106.3 \\
\hline Pm-151 & 1.18 & 88.2 & 11.8 & - & - & - & - & - & - & 91.3 \\
\hline Rh-105 & 1.473333 & 90.2 & 9.8 & - & - & - & - & - & - & 95.1 \\
\hline Ru-103 & 39.35 & 88.0 & 11.4 & 0.0 & - & 0.3 & 0.3 & 0.1 & - & 84.1 \\
\hline Sb-127 & 3.85 & 16.3 & 22.5 & - & 59.5 & - & - & - & 1.6 & 79.7 \\
\hline Sm-153 & 1.9285 & 89.2 & 10.7 & 0.0 & 0.0 & - & - & - & - & 94.4 \\
\hline Sr-91 & 0.400417 & 89.2 & 10.8 & 0.0 & - & - & - & - & - & 103.0 \\
\hline Te-132 & 3.246 & 79.5 & 11.9 & 0.0 & 0.4 & 0.3 & 0.3 & 0.5 & 7.1 & 12.8 \\
\hline U-237 & 6.75 & 86.2 & 13.8 & - & - & - & - & - & - & 69.4 \\
\hline Y-93 & 0.455417 & 100.0 & 0.0 & - & - & - & - & - & - & 110.0 \\
\hline Zr-95 & 64.4 & 98.1 & - & - & 0.3 & 0.2 & 0.1 & - & 1.4 & 91.8 \\
\hline
\end{tabular}

TABLE 5 Partitioning of Decay Products between Process Streams

\begin{tabular}{|c|c|c|c|c|c|c|c|c|c|c|c|c|c|c|}
\hline \multirow[b]{2}{*}{ Isotope } & \multirow[b]{2}{*}{$\begin{array}{c}\text { Half-life } \\
\text { (days) }\end{array}$} & \multirow[b]{2}{*}{ Parent } & \multirow[b]{2}{*}{$\begin{array}{c}\text { Half-life } \\
\text { (days) }\end{array}$} & \multicolumn{9}{|c|}{ Total Activity, $(\mu \mathrm{Ci})$} & \multirow[b]{2}{*}{$\begin{array}{c}\text { Mass } \\
\text { Balance } \\
(\%)\end{array}$} & \multirow[b]{2}{*}{$\begin{array}{c}\text { Analysis } \\
\text { date }\end{array}$} \\
\hline & & & & Feed & Eluent & $\begin{array}{c}1 \mathrm{MHNO}_{3} \\
\text { Wash }\end{array}$ & $\begin{array}{c}\mathrm{H}_{2} \mathrm{O} \\
\text { Wash }\end{array}$ & $\begin{array}{c}\text { Strip } \\
\text { fraction } 1\end{array}$ & $\begin{array}{c}\text { Strip } \\
\text { fraction } 2\end{array}$ & $\begin{array}{c}\text { Strip } \\
\text { fraction } 3\end{array}$ & $\begin{array}{c}\text { Strip } \\
\text { fraction } 4\end{array}$ & Sorbent & & \\
\hline $\mathrm{I}-131$ & 8.02 & Te-131m & 1.25 & $4.2 E+00$ & $2.9 \mathrm{E}+00$ & 3.3E-01 & ND & $2.2 \mathrm{E}-03$ & $\mathrm{BD}$ & $\mathrm{BD}$ & $\mathrm{BD}$ & $6.3 \mathrm{E}-03$ & 78.3 & $5 / 2 / 2014$ \\
\hline La-140 & 1.68 & Ba-140 & 12.79 & $5.9 \mathrm{E}+00$ & $4.5 \mathrm{E}+00$ & $5.4 \mathrm{E}-01$ & ND & $\mathrm{BD}$ & $\mathrm{BD}$ & $\mathrm{BD}$ & ND & ND & 85.8 & $5 / 2 / 2014$ \\
\hline
\end{tabular}




\section{CONCLUSIONS}

Langmuir isotherm parameters for uptake of Mo from $450 \mathrm{gU} / \mathrm{L}$ solution, $1 \mathrm{M} \mathrm{H}^{+}$ solution on S40 sorbent were determined $\mathrm{a}=347, \mathrm{~b}=0.5 \mathrm{mM}^{-1}$. Mo intraparticle diffusivity in $450 \mathrm{~g}-\mathrm{U} / \mathrm{L}$ solution, $1 \mathrm{M} \mathrm{H}^{+}$solution for $\mathrm{S} 40$ sorbent was estimated $D_{p}=7.87 \times 10^{-6} \mathrm{~cm}^{2} / \mathrm{min}$. To enable processing of a target solution obtained from dissolving $225 \mathrm{~g}$ uranium when the volume of solution varies between $0.5 \mathrm{~L}$ and $1 \mathrm{~L}$, alternative process conditions for loading Mo on a 3.5 $\mathrm{x} 5 \mathrm{~cm}$ column were evaluated. It was determined that larger volumes of solution can be loaded under the same process conditions as the combined effects of increased solution volume and higher intraparticle diffusivity have minimal effect on the MTZ.

Irradiated tracer containing uranyl nitrate solution $(899 \mathrm{~g}, \sim 350 \mathrm{~g}-\mathrm{U} / \mathrm{L})$ was loaded onto $3.5 \times 5 \mathrm{~cm}$ column with $\mathrm{S} 40$ sorbent at $25 \mathrm{~mL} / \mathrm{min}$ and $60^{\circ} \mathrm{C}$. The majority of Mo, $98.4 \%$, was recovered in strip fractions \#2 and \#3 (product). The partitioning of actinides, lanthanides, and fission products between different process streams was determined. The majority of activity from other radionuclides, including iodine, actinides, lanthanides, alkaline metal, and transition metal ions, reported to the eluent and acid-wash fractions (waste). Fission products found in the Mo product stream are $<1 \%$ of I-133, Ru-103, Te-132, and Zr-95; 60\% Sb was found in the Mo strip \#1 fraction. 


\section{REFERENCES}

Chung, S. F, and Wen, C. Y. 1968. Longitudinal dispersion of liquid flowing through fixed and fluidized beds. AIChE 14: 857-862.

Jerden, J., Bailey, J., and Vandegrift, G. F. 2014. Ambient Pressure, Acid-Dissolution Front-End Process for Mo-99 Recovery: Final Design and Results from Full-Scale Tests. Argonne National Laboratory report ANL/CSE-14/15.

Krigens, A. J. A. 1968. Compilation of Physical and Chemical Properties of Materials and Streams Encountered in the Chemical Processing Department. Addendum to Harwell report HW-57386.

Marcus, Y. 1997. Ion Properties. New York: Marcel Dekker, p. 259.

Stepinski, D.C., Krahn, E. O., Chung, P.L., and Vandegrift, G. F. 2012. Design of Column Separation Processes for Recovery of Molybdenum from Dissolved High-Density LEU TargetFurther Studies. Argonne National Laboratory report ANL/CSE-13/34.

Wilson E.J., and Geankoplis C.J. 1966. Turbulent liquid phase mass transfer at free gas-liquid interface. I\&EC Fundam. 5: 9-14. 
This page intentionally left blank 



\section{Argonne}

Chemical Sciences and Engineering Division

Argonne National Laboratory

9700 South Cass Avenue, Bldg. 205

Argonne, IL 60439-4837

www.anl.gov

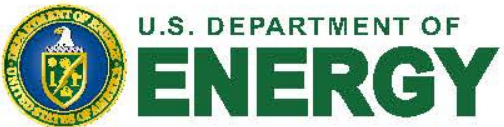

Argonne National Laboratory is a U.S. Department of Energy

laboratory managed by UChicago Argonne, LLC 